Journal of the Magnetics Society of Japan Vol. 15 Supplement, No. S2 (1991)

(C) 1991 by The Magnetics Society of Japan

\title{
MAGNETIC AND STRUCTURAL PROPERTIES OF COCr DEPOSITED ON A VARIETY OF FLEXIBLE SUBSTRATES
}

\section{J. SKORJANEC, J. CLOSE, P. IVERSON, V. COTTLES, and D. DAHLBERG*}

3M Corporation, Bldg. 201-1N-34, St. Paul, MN 55144

*Also in the School of Physics and Astronomy, U. of Mn

Abstract-- A variety of flexible substrates have been utilized for the preparation of $\mathrm{CoCr}$ thin films. It has been found that all the substrates used, except the high modulus film, allow the production of high quality $\mathrm{CoCr}$ films. As determined by their bulk magnetic properties, their saturation magnetization, anisotropy energy, and hysteresis loops, most of the films would be adequate for magnetic recording media. The primary structural characterization probe was $\mathrm{x}$-ray scattering. As expected, the perpendicular magnetic properties of the films were correlated with the measured degree of crystalline orientation as determined by the $x$-ray scattering.

\section{INTRODUCTION}

The development of flexible thin film magnetic media for perpendicular recording requires high quality thin magnetic films to be grown on flexible films or substrates. In this development process, it is important to not only find candidates for the magnetic thin film, it is also necessary to match the magnetic film to a substrate. This process requires a magnetic candidate to be identified and then prepared on a variety of substrates. The successful preparation on a large number of substrates expands the processes available for providing flexible media.

For these reasons, we have undertaken a study of a number of flexible substrates which have been considered for metal film magnetic recording tape. Thin films of $\mathrm{CoCr}$ were chosen as the magnetic media because of its superior magnetic properties for perpendicular recording $[1,2]$ and its corrosion resistance [3]. Although the films in the present study were not used in magnetic recording tests, the bulk magnetic measurements made indicate that films prepared on the different types of substrates could perform as recording media. In the following section, the enumeration of the substrates and the deposition details are discussed. The experimental section which follows, details the magnetic and structural measurements used in this investigation. The experimental results section details the findings of this study and is followed by our conclusions.

\section{SAMPLE PREPARATION}

As mentioned above, a number of different substrates were investigated. They are polyimide, polyester, polysulfone, polyetherimide, polycarbonate, polyphenyloxide, polyethersulfone, high modulus films (HMF), and D-100. To provide good control of film properties, sputtering was used for deposition. Both RF and DC magnetron films were deposited on five of the substrate candidates to determine deposition process dependence. After determining that high quality films could be prepared by either deposition process, the remaining four substrates had films deposited by only one process; three with if sputtered films and one with dc magnetron sputtered films.

The sputtering system was a cryopumped Perkin-Elmer 24008L deposition system, with a base pressure lower than 1x10-7 Torr. During sputtering, an argon pressure was maintained in the range of 1-5 mTorr. The deposition typically occurred at rates of $20 \mathrm{~nm} / \mathrm{min}$. to $500 \mathrm{~nm} / \mathrm{min}$ with substrates stationary. A roll coater of proprietary design was used to produce $\mathrm{CoCr}$ films from targets of different composition on the substrates. For this, the deposition rate was typically $500 \mathrm{~nm} / \mathrm{min}$ with web speeds up to $24 \mathrm{in} / \mathrm{min}$. The primary variable used to control 


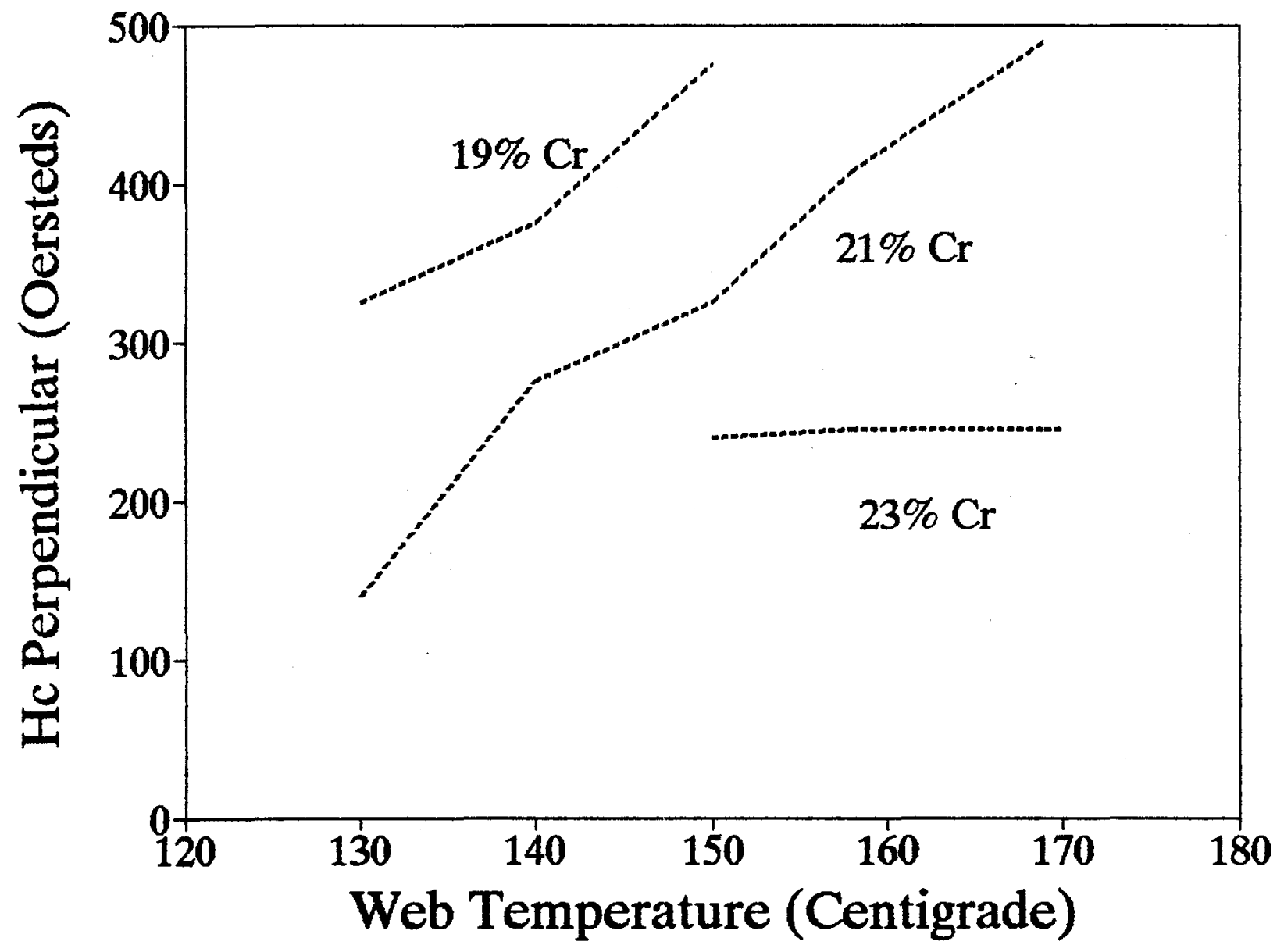

Figure 1. The perpendicular coercivity as a function of web or substrate temperature for three different $\mathrm{Cr}$ concentrations. The substrate used for the data in this figure was polyester.

the growth of the magnetic films was the web or substrate temperature. As an example of the variability of the film parameters variation with growth temperature, figure 1 exhibits the measured coercivity of three different $\mathrm{CoCr}$ compositions as a function of the web temperature. The substrate used for these data was polyester.

\section{EXPERIMENTAL PROCEDURES}

The magnetic properties investigated include the perpendicular anisotropy energy (Hk), the saturation magnetization (Ms), the perpendicular and parallel coercivities (Hc perp and Hc para), and the perpendicular and parallel remanent magnetizations (Mr perp and Mr para). These data were taken on a conventional vibrating sample magnetometer with magnetic field rates of $2 \mathrm{kOe} / \mathrm{min}$ sweeps. In addition to the magnetic properties, the structural properties of the films were monitored by $x$-ray diffraction. By tuning the deposition process, all films were made to exhibit a similar preferential growth direction as determined by rocking curve data (dTheta 50 ).

\section{EXPERIMENTAL RESULTS}

In discussing the results, it is maybe most instructive to note that for perpendicular recording 


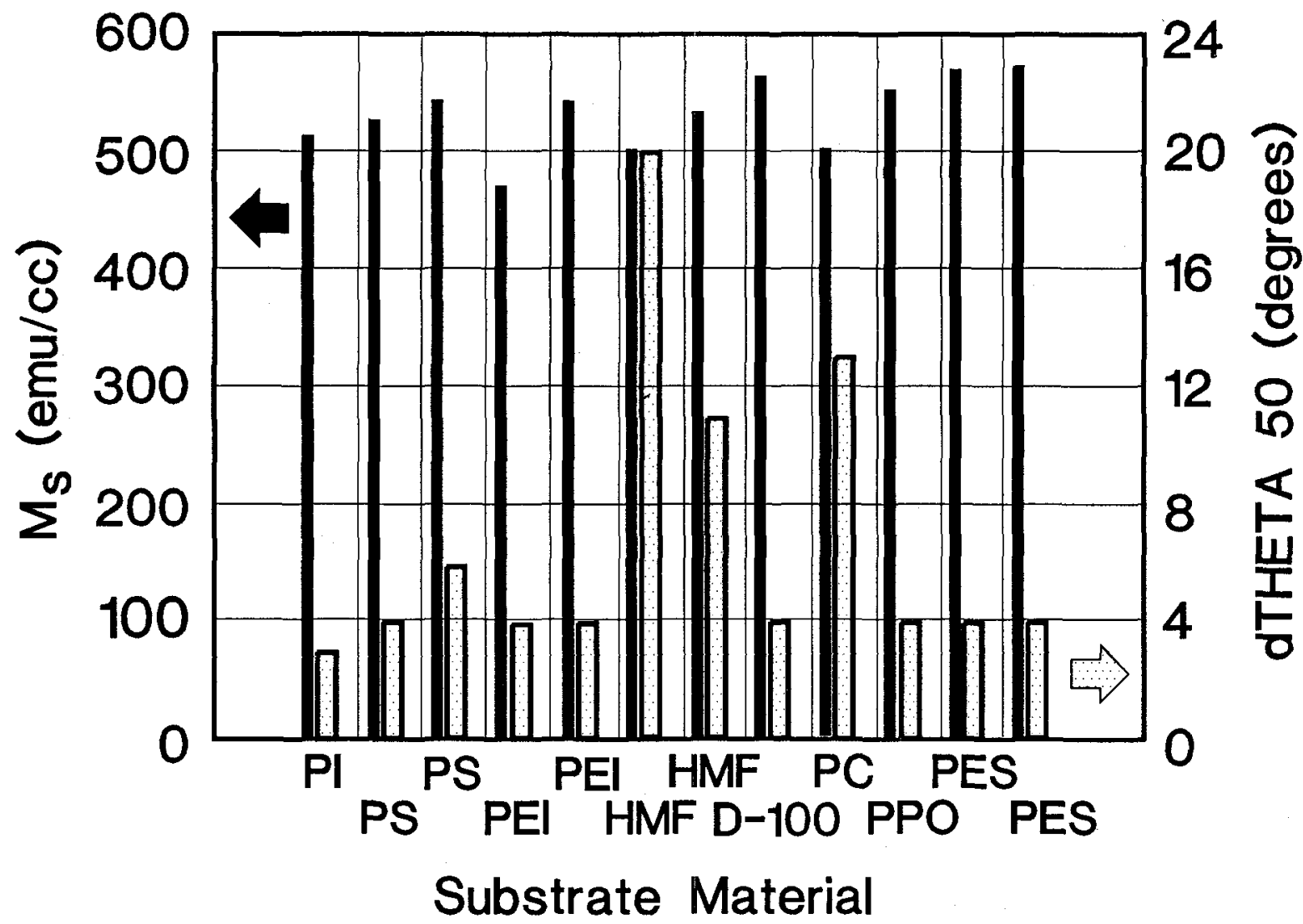

Figure 2. The saturation magnetization and the degree of alignment as measured by the delta theta 50 rocking curve $\mathrm{x}$-ray spectra for the substrates used in this study. The substrates are identified as follows: PI (polyimide), PS (polysulfone), PEI (polyetherimide), HMF (high modulus films), D-100, PC (polycarbonate), PPO (polyphenyloxide), and PES (polyethersulfone). Where a substrate is listed twice, the first listing is for RF sputtered films and the second is for DC sputtered films.

media, a measure of the degree of alignment is most instructive. In addition, a measure of the quality of the magnetic material is the saturation magnetization. Exhibited in figure 2 is both the degree of alignment, dTheta 50 , and the saturation magnetization, Ms, for the substrates reported here.

Three comments should be made about the data exhibited in this figure. First, for all the substrates, the saturation magnetizations for the "tuned" films were within $+1-5 \%$ of each other. Next, the growth quality of the films, with only two exceptions, were outstanding. For those two substrates, the high modulus films and the polycarbonate, the rocking curves indicated poor orientational growth. Third, it is very interesting to note that, in fact, the film growth orientation and the saturation magnetization appear to be unrelated. This last feature may be interpreted as indicating that poor growth alignment does not alter the magnetic structure of the film, i.e., the same crystal structure is present but with a random orientation. This conclusion is also borne out by the $x$-ray diffraction patterns of the films. These show the same crystal structure within experimental error for all the films, independent of substrate. 
As shown in figure 2, all of the substrates with the exception of polycarbonate and the high modulus films, were able to support magnetic films with a very high degree of alignment as measured by the dTheta $50 \mathrm{x}$-ray patterns. It has been determined that the other properties which one might associate with a perpendicular magnetic recording medium, such as the perpendicular anisotropy energy, Hk, the coercivity, $\mathrm{Hc}$ and the perpendicular/parallel remanence ratio, Mr perp/ Mr para, do not seem to be sensitive to dTheta 50. The best relationships between the various properties mentioned above can be stated as follows: first, there is a correlation, roughly linear, between $\mathrm{Hk}$ and both $\mathrm{Hc}$ and $\mathrm{Mr}$ perp/Mr para. Also, there is a minimum dTheta $\mathbf{5 0}$ necessary for high Hk films. The data in figures 3,4 ,and 5 substantiate these statements.

Figure 3 shows an effective threshold for high perpendicular anisotropy energies to be films with dTheta 50's of less than approximately 5 degrees. These data come from the best films but in general this result is born out. Figures 4 and 5 exhibit the relationships between the Hc and the Mr Perp/ Mr para. As shown in these figures, the bulk magnetic properties appear to scale with the perpendicular anisotropy energy and not depend upon the saturation magnetization and only indirectly with the degree of alignment of the films.

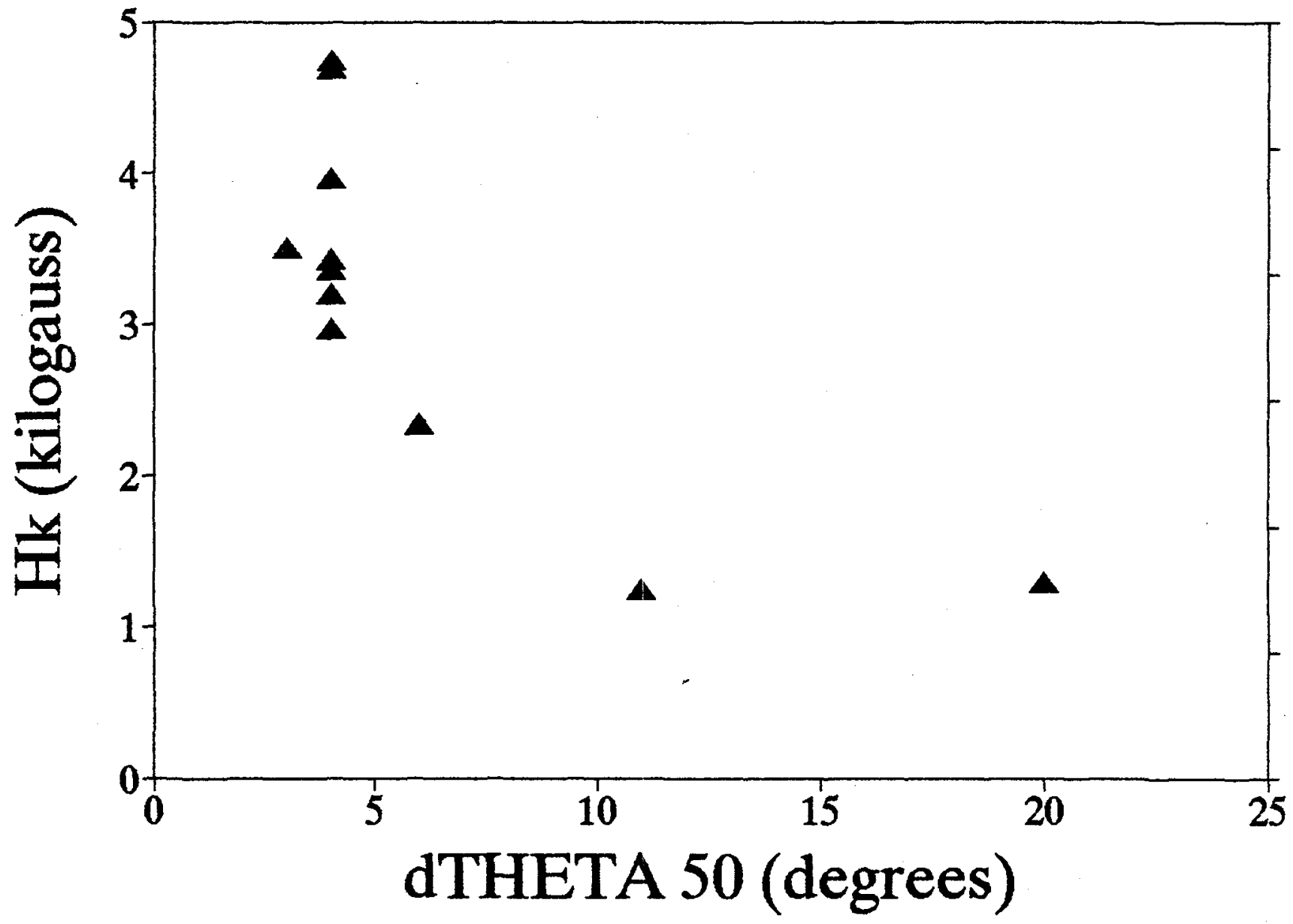

Figure 3. A comparison of the measured perpendicular anisotropy energies and the delta theta 50 rocking curve $x$-ray data for various substrates. Notice the threshold behavior of the anisotropy energy. 


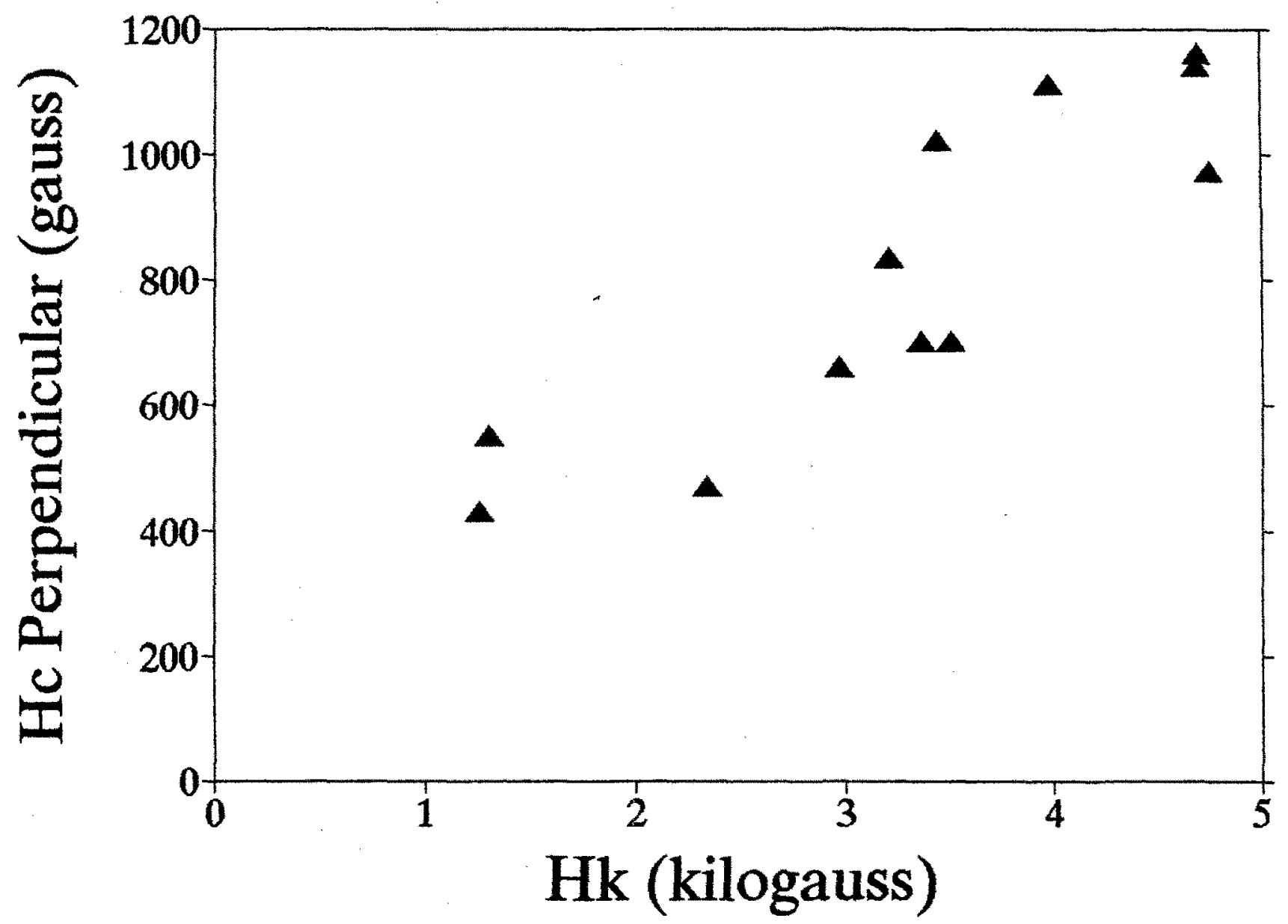

Figure 4. The perpendicular coercivity plotted against the anisotropy energies measured. These data come from a mixture of substrates used in this study. 


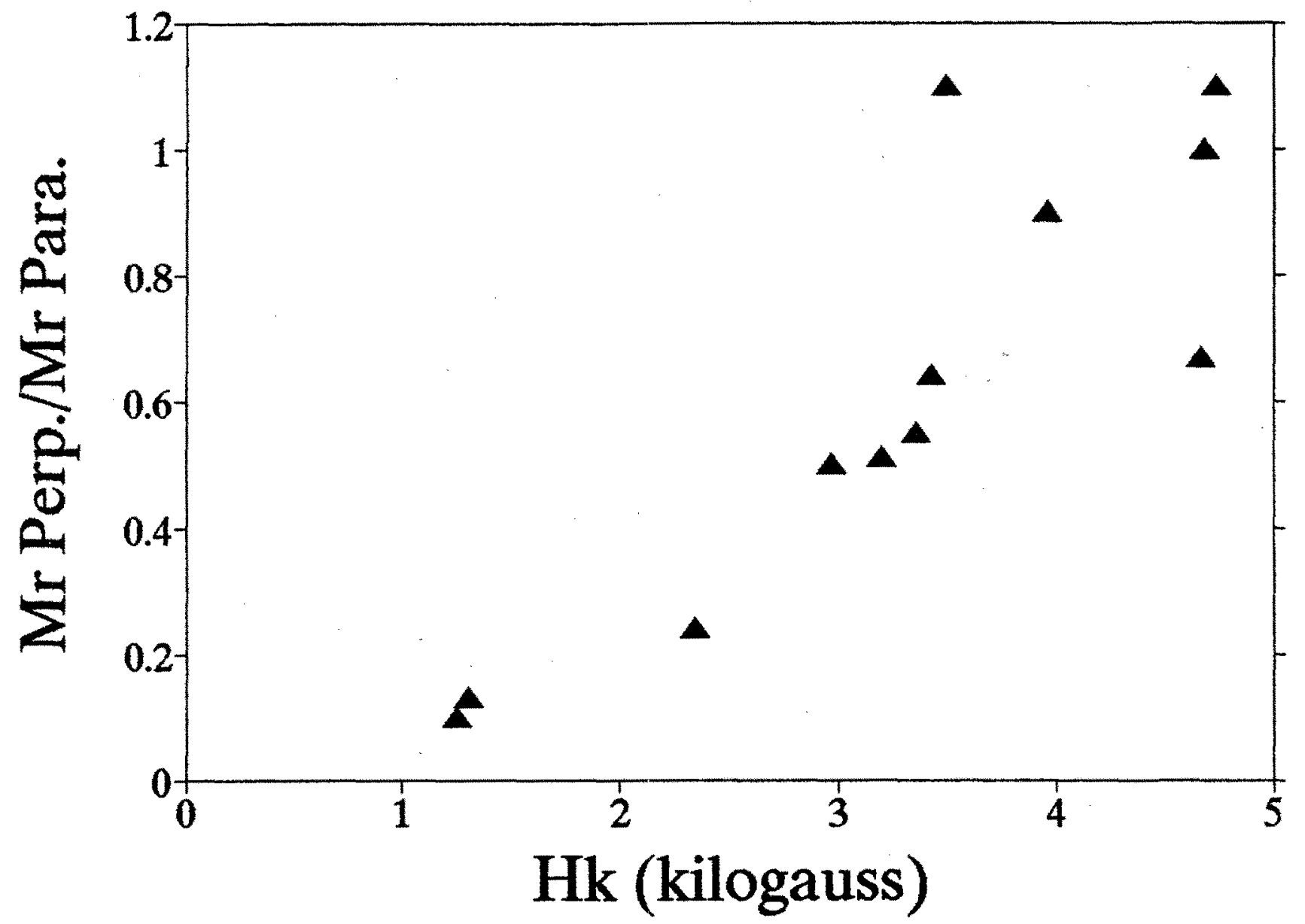

Figure 5. A comparison of the ratio of perpendicular remanent magnetization to the parallel remanent magnetization versus the anisotropy energy. These data come from a mixture of all the substrates studied in this investigation.

\section{SUMMARY}

A study of the deposition of CoCr thin films on a variety of substrates has been undertaken. With only two substrate exceptions, high modulus films and polycarbonate, magnetic films have been produced with a high degree of alignment as measured by $x$-ray rocking curves. The saturation magnetization of the films is found to be independent of alignment of the films. The correlation of the alignment with the perpendicular anisotropy energies measured appears to be a threshold, i.e., the delta theta 50 must be less than 5 degrees in order to obtain anisotropy energies greater than $2500 \mathrm{Oe}$. It is maybe more

surprising to find that an approximate linear relationship exists between the anisotropy energy and both the coercivity and the remanent perpendicular magnetization. This implies both the coercivity and the remanent magnetization have the same threshold dependence on the degree of film alignment as does the anisotropy energy.

\section{REFERENCES}

[1] S.Iwasaki and K. Takemura, IEEE Trans Magn. MAG-11,1173 (1975).

[2] S. Iwasaki, K. Ouchi, and N. Honda, IEEE Trans. Magn. MAG-16, 1111 (1980).

[3] G.L. Mcintire and C.F. Brucker, IEEE Trans. Magn. 24, 5 (1988). 